\section{A prospective ultrasound biomicroscopy evaluation of changes in anterior segment morphology following laser iridotomy in European eyes}

K Mansouri, ND Burgener, M Bagnoud and T Shaarawy
Glaucoma Sector, Ophthalmology Clinic, University of Geneva, Geneva, Switzerland

Correspondence: K Mansouri, Glaucoma Sector, Ophthalmology Clinic, University of Geneva, 22, rue Alcide, Jentzer 1211

Genève, Switzerland Tel: + 41223828 400; Fax: + 41223828382

E-mail: kawehm@ yahoo.com

Received: 16 June 2008 Accepted in revised form: 28 November 2008 Published online: 9 January 2009

Financial interest: None

\section{Abstract}

Purpose The aim of this study is to quantify changes in anterior segment morphology by the use of ultrasound biomicroscopy (UBM) after Nd:YAG laser iridotomy in primary angle closure (PAC) and PAC glaucoma (PACG) in European eyes.

Methods A total of 35 eyes of 28 consecutive patients presenting with PAC or PACG were examined by UBM at presentation, and 1 week after Nd:YAG laser peripheral iridotomy (LPI). Mean age of patients was $63.5 \pm 14.4$ (SD) years. Seventeen patients were females $(60 \%)$. The trabecular-iris angle (TIA) was measured in the superior, nasal, inferior, and temporal quadrants. Baseline measurements were made under light and dark conditions. Main outcome measure UBM measurements of the trabecular-iris angle (TIA) and the angle opening distance (AOD).

Design Consecutive observational case series.

Results All measurements were made in four quadrants. Before LPI, mean superior TIA was $3.59 \pm 4.5^{\circ}$ (mean \pm SD), nasal TIA was $6.37 \pm 4.9^{\circ}$, inferior TIA was $9.33 \pm 7.6^{\circ}$, and temporal TIA was $8.65 \pm 7.3^{\circ}$ in light conditions. After LPI, these values increased, respectively, to $12.58 \pm 6.9^{\circ}(P<0.05)$, $15.40 \pm 6.8^{\circ}(P<0.05), 16.37 \pm 7.4^{\circ}(P<0.05)$, and $15.95 \pm 11.3^{\circ}(P<0.05)$, showing a significant widening of the angle in all four quadrants. Superior AOD increased from $0.060 \pm 0.07$ to
$0.107 \pm 0.07 \mathrm{~mm}(P=0.09)$. No serious LPI-related complications were encountered. Conclusions Dimensions of the anterior chamber angle can be significantly influenced by Nd:YAG laser iridotomy in narrow angle European eyes. UBM examination is a viable tool for the quantitative evaluation of the anterior chamber angle before and after laser iridotomy.

Eye (2009) 23, 2046-2051; doi:10.1038/eye.2008.395; published online 9 January 2009

Keywords: angle closure; glaucoma; laser peripheral iridotomy; ultrasound

biomicroscopy

Introduction

Primary angle closure (PAC) glaucoma (PACG) is considered to be a relatively uncommon entity in Caucasian populations. It is the major form of glaucoma and a significant cause of blindness in East and South Asia. ${ }^{1-3}$ It is an aggressive form of glaucoma and may be associated with higher rates of blindness in Asia when compared with primary angle open glaucoma (POAG) ${ }^{4,5}$

Nd:YAG laser peripheral iridotomy (LPI) is recognized as the first-line intervention for preventing the progression of PAC to PACG and for preventing recurrent attacks. ${ }^{6,7}$ It has also been proposed as a preventive measure in predisposed fellow eyes. ${ }^{8,9}$ The mechanism of 
LPI is the elimination of iris convexity secondary to pupillary block and subsequent reversing of appositional angle closure. These anatomical and morphological changes are difficult to evaluate by slit-lamp biomicroscopy. Currently, gonioscopy is the reference standard for clinical assessment of angle-closure. The main limitations of gonioscopic studies are inter and intraobserver bias. ${ }^{10}$ Furthermore, in cases of iris plateau configuration, gonioscopy provides only limited information on the degree of angle opening. Ultrasound biomicroscopy (UBM) allows reproducible imaging of the cross-sectional anterior chamber (AC) anatomy as well as quantitative measurements of the anterior segment in a semiautomated way. ${ }^{11}$ It is, however, a relatively difficult technique to perform, and is also subject to inter and intraobserver bias.

It has been argued that angle closure glaucoma has distinct differences between European and Asian patients. ${ }^{12}$ One finding is that central AC depth is shallower in East Asians than in Europeans, although this is not universally accepted. ${ }^{13}$ Meaningful comparison between studies is currently hindered by differences in patient selection, examination technique, and case definition. Most available data on angle closure glaucoma stems from research in Asian patients. However, PAC and PACG are also present in Europeans and might be under- or misdiagnosed as POAG due to low prevalence and resulting lack of familiarity in general ophthalmologists.

One scientific deficiency is the paucity of prospective data providing insight into the natural history and in the response to treatment in European patients. The effect of LPI in European eyes has only been investigated in one UBM study. ${ }^{14}$ Furthermore, the current World Glaucoma Association Consensus Statement on angle closure and angle closure glaucoma calls for more research to determine ethnic variations in response to iridotomy. ${ }^{15}$ The aim of this study is to quantify changes in anterior segment morphology by the use of UBM after Nd:YAG laser iridotomy in European patients with PAC and PACG in light and dark conditions.

\section{Materials and methods}

In all, 35 eyes of 28 consecutive patients presenting with PAC (occludable drainage angle and features of trabecular obstruction, such as peripheral anterior synechiae (PAS), iris whorling, glaukomflecken, excessive pigment deposition on the trabeculum, and/or raised IOP) or PACG (PAC together with signs of glaucomatous optic neuropathy) at our tertiary referral centre were examined with UBM at presentation, and 1 week after Nd:YAG LPI. The diagnosis was based on clinical history, slit-lamp biomicroscopy, and visual field testing, and in accordance with the definition of Foster et al. ${ }^{16}$ Patients with the evidence of PAS of more than $90^{\circ}$ were excluded. Five eyes presented with acute angle closure and four eyes were referred to our clinic with chronic angle closure, the remainder were recruited at the clinic. The decision to perform LPI was taken by the attending physician who was one of the study authors (KM).

Baseline measurements were made under light and dark conditions by two experienced examiners with good interobserver agreement $(\kappa=0.79)$. Measurement of the angle was made before and after LPI using the UBM software (C 1, 1999). UBM examinations were performed with the UBM Model 840 machine (Paradigm medical Industries Inc., USA) and a 50-MHz transducer probe. After topical anaesthesia with $0.4 \%$ oxybuprocaine in both eyes, a 22- or 24-mm eyecup was applied and filled with $2 \%$ methylcellulose as a coupling agent. The scleral spur was localized by the operator. Pavlin et al ${ }^{17}$ and Ishikawa et $a l^{11}$ have described different UBM parameters that characterize the $\mathrm{AC}$ angle. The trabecular iris angle (TIA) was measured with the apex at the iris recess and the arms passing through the point on the meshwork $500 \mu$ from the scleral spur and the point on the iris perpendicularly opposite. The angleopening distance (AOD) as the distance between the posterior corneal surface and the anterior iris surface was measured on a line perpendicular to the trabecular meshwork at $500 \mu \mathrm{m}$ from the scleral spur. The scleral spur was identified based on the differential tissue density between the collagen fibres of the scleral spur and the longitudinal muscle of the ciliary body (Figure 1).

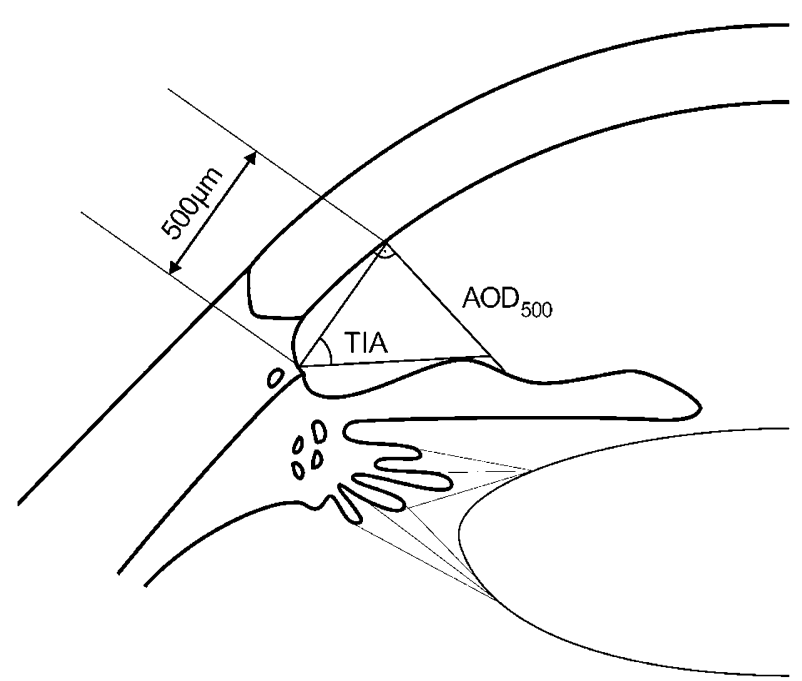

Figure 1 Schaematic representation of the anterior segment measurements by UBM. Trabecular iris angle (TIA) and angleopening distance (AOD) at $500 \mu \mathrm{m}$ anterior to the scleral spur are indicated. 
The LPI was performed using a Nd:YAG laser, set at variable energy levels between 2 and $6 \mathrm{~mJ}$ (5-10 shots). All patients received the same preinterventional regimen that consisted of three drops of pilocarpine $2 \%$ half an hour before the intervention and one drop of brimonidine. Using an Abraham lens, one opening was performed selecting, if possible, a crypt in the peripheral superotemporal or superonasal iris. All iridotomies were confirmed to be patent using UBM. After the intervention, patients were given one drop of brimonidine and prednisolone eye drops (q.i.d) for 7 days. All patients gave written informed consent to be included in this study. The study adhered to the tenets of the Declaration of Helsinki.

On the basis of a previously published study, the minimum sample size to detect an increase of AOD 500 by $40 \mu \mathrm{m}$ with a standard deviation of $70 \mu \mathrm{m}$, for a study with a power of $90 \%$ at a significance level of $5 \%$, was calculated to be 33 eyes. ${ }^{6}$ Student's paired $t$-test was used to analyse the UBM parameters and a $P$-value of less than 0.05 was considered statistically significant. To investigate the potential effects of including both eyes of a subject in the study sample, the overall analysis was performed with and without the seven contralateral eyes. The results were similar and statistically not significant in both situations, and therefore we describe the results for all 35 eyes. Data were prospectively collected on data sheets at each visit. The statistical analysis was performed using STATA 9.0.

\section{Results}

All measurements were made in the superior, nasal, inferior, and temporal quadrants at each examination in both light and dark conditions. A total of 35 eyes of 28 European patients (all of Caucasian ethnicity) with PAC or PACG were enrolled consecutively in the study and seen at 1-week follow-up. Seven patients required bilateral LPI and both eyes were included in the analysis. Average age of patients was 63.5 years $(\mathrm{SD}=14.4$; range $=30-83$ years). There were 17 females $(60 \%)$ and 11 male patients (40\%). In all, 19 eyes had PAC and 16 eyes had PACG, out of which five were acute angle closures. LPI was carried out in the superotemporal quadrant in 26 eyes $(74 \%)$ and in the superonasal quadrant in nine eyes $(26 \%)$.

Changes in angle dimensions after LPI were seen both in light and dark conditions. Under light conditions, the mean TIA for all four quadrants increased significantly from $6.98 \pm 4.6^{\circ}$ to $15.05 \pm 6.8^{\circ}$ after LPI $(P<0.05)$. The mean superior AOD increased from $0.060 \pm 0.07$ to $0.107 \pm 0.07 \mathrm{~mm}(P=0.09)$, and the mean inferior AOD increased from $0.100 \pm 0.10$ to $0.152 \pm 0.08 \mathrm{~mm}$ after LPI $(P=0.148)$, but these values did not reach statistical
Table 1 UBM parameters in all quadrants before and after laser peripheral iridotomy (LPI) in light conditions

\begin{tabular}{lccc}
\hline UBM parameter & $\begin{array}{c}\text { Pre-LPI } \\
(\text { mean } \pm S D)\end{array}$ & $\begin{array}{c}\text { Post-LPI } \\
(\text { mean } \pm S D)\end{array}$ & P-value \\
\hline Superior TIA (degrees) & $3.59 \pm 4.5$ & $12.58 \pm 6.9$ & $<0.05$ \\
Superior AOD500 (mm) & $0.060 \pm 0.07$ & $0.107 \pm 0.07$ & $0.09^{\mathrm{a}}$ \\
Nasal TIA & $6.37 \pm 4.9$ & $15.40 \pm 6.8$ & $<0.05$ \\
Inferior TIA & $9.33 \pm 7.6$ & $16.37 \pm 7.4$ & $<0.05$ \\
Inferior AOD500 & $0.100 \pm 0.10$ & $0.152 \pm 0.08$ & $0.14^{\mathrm{a}}$ \\
Temporal TIA & $8.65 \pm 7.3$ & $15.95 \pm 11.3$ & $<0.05$ \\
\hline
\end{tabular}

AOD500 $=$ angle-opening distance at $500 \mu \mathrm{m}$ from the scleral spur; TIA = trabecular iris angle; $\mathrm{UBM}=$ ultrasound biomicroscopy.

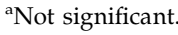

Table 2 UBM parameters in all quadrants before and after laser peripheral iridotomy (LPI) in dark conditions

\begin{tabular}{lccc}
\hline UBM parameter & $\begin{array}{c}\text { Pre-LPI } \\
(\text { mean } \pm \text { SD })\end{array}$ & $\begin{array}{c}\text { Post-LPI } \\
(\text { mean } \pm S D)\end{array}$ & P-value \\
\hline Superior TIA (degree) & $3.09 \pm 5.7$ & $7.38 \pm 6.3$ & $<0.05$ \\
Superior AOD500 (mm) & $0.049 \pm 0.08$ & $0.105 \pm 0.07$ & $<0.05$ \\
Nasal TIA & $5.21 \pm 6.1$ & $10.26 \pm 7.5$ & $<0.05$ \\
Inferior TIA & $5.39 \pm 6.3$ & $10.21 \pm 8.9$ & $<0.05$ \\
Inferior AOD500 & $0.056 \pm 0.11$ & $0.137 \pm 0.13$ & $0.06^{\mathrm{a}}$ \\
Temporal TIA & $6.10 \pm 5.4$ & $12.00 \pm 9.1$ & $<0.05$ \\
\hline
\end{tabular}

AOD500 $=$ angle-opening distance at $500 \mu \mathrm{m}$ from the scleral spur; TIA = trabecular iris angle; $\mathrm{UBM}=$ ultrasound biomicroscopy .

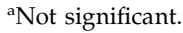

significance. Under dark conditions, the mean TIA for all four angles was $4.95 \pm 4.16^{\circ}$ before and $9.81 \pm 5.65^{\circ}$ after LPI, showing a significant increase in angle opening $(P=0.001)$. Mean superior AOD increased from $0.049 \pm 0.08$ to $0.125 \pm 0.07 \mathrm{~mm}(P=0.01)$ and the mean inferior AOD increased from $0.056 \pm 0.11$ to $0.138 \pm 0.13 \mathrm{~mm}$ after LPI $(P=0.06)$, but this increase did not reach statistical significance. Tables 1 and 2 show the precise changes in UBM parameters after LPI. Table 3 shows changes in UBM parameters going from light to dark conditions before and after LPI.

UBM verification of patency of LPI was carried out in all study patients, showing all openings to be patent and of adequate size. The number of eyes classified as occludable (three quadrants with iridotrabecular contact) decreased by $63 \%$ (30 eyes before, eight after LPI). Figure $2 \mathrm{a}$ and $\mathrm{b}$ show UBM scans of an angle before and after LPI.

\section{Discussion}

The aim of this study was to quantify the anterior segment changes in European patients after LPI. The 
Table 3 Changes in UBM parameters going from light to dark before and after laser peripheral iridotomy

\begin{tabular}{llccc}
\hline UBM parameter & $\begin{array}{c}\text { Pre-LPI } \\
\text { (mean) }\end{array}$ & P-value & $\begin{array}{c}\text { Post-LPI } \\
\text { (mean) }\end{array}$ & P-value \\
\hline Superior TIA (degrees) & 0.50 & $0.682^{\mathrm{a}}$ & 5.20 & $<0.05$ \\
Superior AOD 500 (mm) & 0.012 & $0.671^{\mathrm{a}}$ & -0.018 & $0.533^{\mathrm{a}}$ \\
Nasal TIA & 1.16 & $0.476^{\mathrm{a}}$ & 5.14 & $<0.05$ \\
Inferior TIA & 3.94 & $0.063^{\mathrm{a}}$ & 6.16 & $<0.05$ \\
Inferior AOD 500 & 0.045 & $0.240^{\mathrm{a}}$ & 0.015 & $0.905^{\mathrm{a}}$ \\
Temporal TIA & 2.55 & $0.168^{\mathrm{a}}$ & 3.95 & $0.223^{\mathrm{a}}$ \\
\hline
\end{tabular}

AOD500 $=$ angle-opening distance at $500 \mu \mathrm{m}$ from the scleral spur; TIA = trabecular iris angle; UBM = ultrasound biomicroscopy. ${ }^{a}$ Not significant.

results of this study confirm earlier reports on increased AC angle opening following LPI in different populations. ${ }^{6,8,9,18}$

In the past 25 years, Nd:YAG iridotomy has replaced surgical iridectomy as the mainstay of PAC treatment.

However, despite its frequency of use, few studies have evaluated its effect on AC morphology in a prospective and reproducible manner. This was mainly due to the sole availability of gonioscopy as an assessment tool. This technique is limited by its subjectivity and relative difficulty. With the advent of UBM, detailed and objectively quantifiable imaging of the anterior segment morphology has become possible. Although UBM has been commercially available since 1990, it has not become part of the routine clinical practice. This is most likely due to its time-consuming nature, contact with the globe, and difficulty in manipulation. It is argued that it can artifactually widen the angle by inadvertent corneal indentation and performing in the supine position. Only one paper has evaluated UBM changes after LPI in European patients, whereas all other studies were performed on Asian populations who generally suffer from a higher prevalence of narrow angles. ${ }^{6-9,14}$

We found that TIA and $\mathrm{AOD}_{500}$ both increased after LPI in different light conditions. Our study showed that LPI was effective in reducing the number of eyes classified as occludable by $63 \%$. Gazzard et al $^{6}$ reported a corresponding rate of $40 \%$ in an Asian population. In our series, mean TIA significantly increased from $6.98 \pm 4.6^{\circ}$ to $15.05 \pm 6.8^{\circ}$ under light conditions $(P<0.05)$. This compares well with Marraffa et $\mathrm{al}^{14}$ who studied a group of Italian PACG patients and showed that the TIA nearly doubled from $10.69 \pm 8.88^{\circ}$ to $21.03 \pm 11.28^{\circ}$. Contrary to our series, they included patients with more than $90^{\circ}$ PAS. We excluded patients with more than $90^{\circ}$ PAS from our cohort, as this group could be expected to show reduced opening of the AC angle after LPI as is supported by three recent studies. ${ }^{7,8,19}$ Despite our strict exclusion of PAS cases, our baseline measurements for
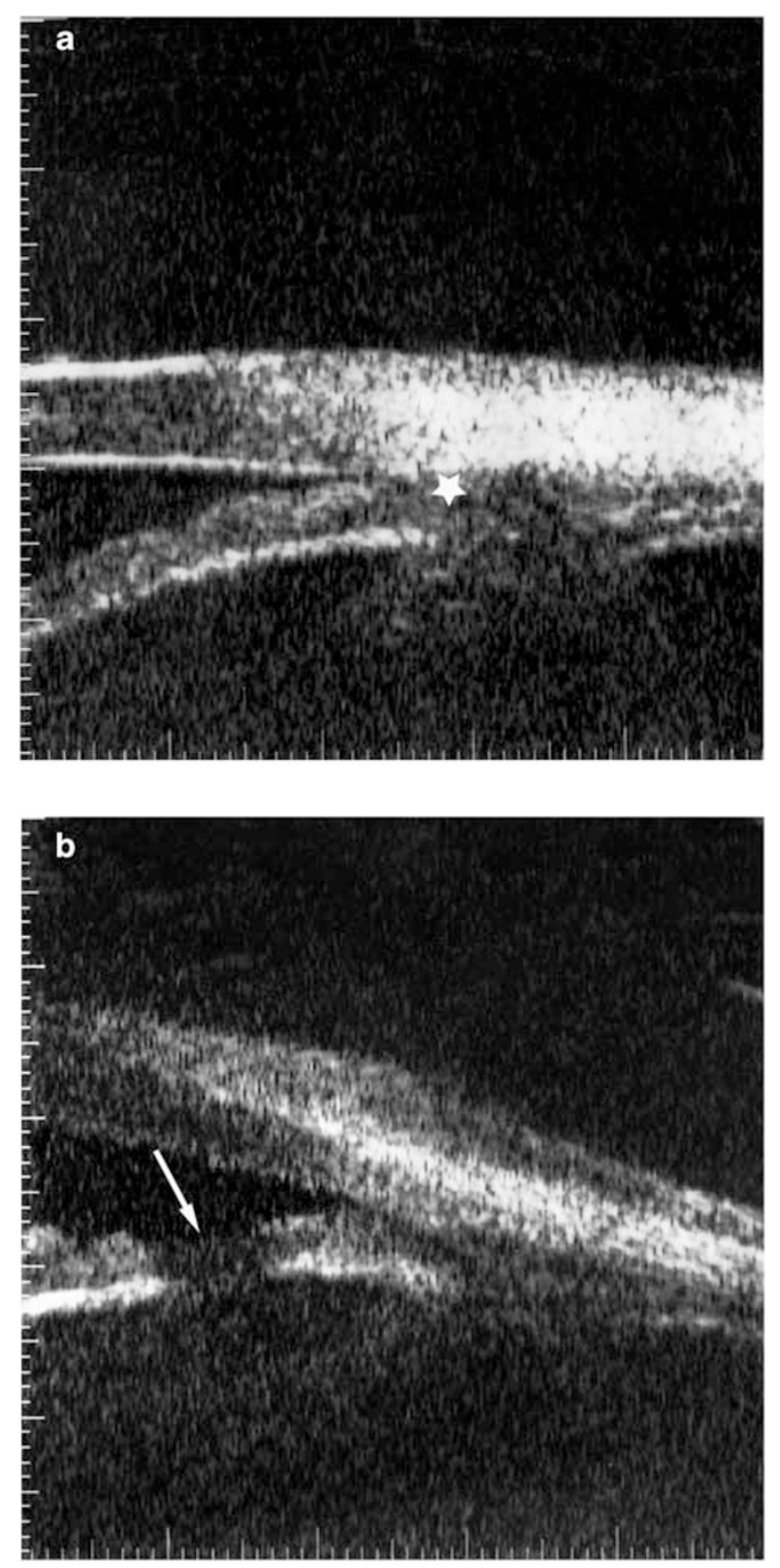

Figure 2 (a and b) Cross-sectional UBM scans of an angle before (a) and after (b) laser peripheral iridotomy (LPI). Note the flattening of the convex iris and widening of the angle following LPI in an eyes with primary angle closure. The scleral spur is indicated by a star (a) and the iridotomy by an arrow (b).

TIA and AOD were mostly smaller than in other studies. We have no valid explanation for this finding. We also did not include IOP and other clinical parameters as an outcome measure, as this study only investigated morphological changes in AC angle.

In recent years, increased efforts have been put into documenting the effects of laser iridotomy by objective methods. Two recent papers by Dada $e t \mathrm{al}^{8}$ and Kaushik et $a l^{18}$ quantified changes in anterior segment 
morphology after LPI by UBM in Indian patients. Both studies confirm significant widening of the AC angle.

Kaushik et $a l^{18}$ included 55 patients with PACG and PAS of less than $180^{\circ}$ and measured changes in AOD at 250 and $500 \mu \mathrm{m}$. They found a mean increase of $\mathrm{AOD}_{500}$ at the LPI quadrant from $0.112 \pm 0.08$ to $0.170 \pm 0.08 \mathrm{~mm}$, an increase of $71.2 \%(P<0.05)$. Dada et $a l^{8}$ included a mixed group of 93 patients with PAC and PACG presenting more than $180^{\circ}$ PAS. This group had an $\mathrm{AOD}_{500}$ increase of $0.107 \pm 0.06-0.208 \pm 0.18 \mathrm{~mm}$ (PAC; $P<0.001)$ and $0.065 \pm 0.09-0.076 \pm 0.13 \mathrm{~mm}$ (PACG; $P=$ NS). These results compare well with our series in which the mean superior $\mathrm{AOD}_{500}$ increased from a mean of $0.060 \pm 0.07-0.107 \pm 0.07 \mathrm{~mm}(P=0.09)$ in light and $0.049 \pm 0.08-0.105 \pm 0.07$ in dark conditions $(P<0.05)$. The fact that $\mathrm{AOD}_{500}$ unexpectedly did not significantly change in all quadrants might be due to a small sample size, although this study was adequately powered to detect a change of $40 \mu \mathrm{m}$.

The changes in the anterior angle chamber that were observed in this study are consistent with the theory that the iridotomy hole influences the aqueous dynamic and reduces the pressure gradient between the anterior and posterior part of the AC. This translates into iris shape changing from steep convex to a more flattened one as well as the entire iris falling back. Interestingly, the LPI quadrant opened more than the opposite quadrant. Although this increase was more accentuated in light conditions than under darkness, it is in accordance with Kaushnik et $a l^{18}$ who found a mean gonioscopy grade increase of 1 in the LPI quadrant compared to 0.11 in the opposite one. We, therefore, concur with their explanation that this change could be due to more constant percolation of aqueous through the iridotomy.

With the benefits of LPI thus well established, the right timing of the intervention as well as influencing factors still remain a matter of controversy and could be the subject of longitudinal studies. Although one report purported that the presence of established glaucoma per se did not appear to negatively influence the results, ${ }^{18}$ two others have suggested that once PACG is manifest, LPI may be of limited success in lowering IOP. ${ }^{5,7}$ More research would be desirable to confirm these findings.

Anterior segment optical coherence tomography (AS-OCT) has recently been introduced as an alternative imaging modality to UBM. ${ }^{20}$ It has a potential advantage over UBM in that it does not require a water bath. Several studies have evaluated quantitative measurement of the AC angle using AS-OCT. ${ }^{21}$ A shortcoming of AS-OCT is the fact that the pigmented posterior layer of the iris prevents infra-red light transmission beyond this structure and the ciliary body and suprachoroidal space are not visualized.
A limitation of the method is that the UBM software requires the operator to define the position of the scleral spur, which then serves as an anatomic landmark from where the UBM measurements are calculated. Variations in reflectivity signals may not always give a sharp contrast to accurately determine the position of the scleral spur, which can cause inconsistent localization of this reference point. The manual and subjective identification of the scleral spur may introduce errors in the measurements. In addition, because of irregularities in the iris surface, AOD is not always a reproducible index for the angle. Therefore, this technique is subject to variability in intra and interobserver reproducibility. ${ }^{22,23}$ The fact that seven patients had both eyes included might be a small limitation of this study. However, when we performed the analysis with and without the contralateral eyes, this did not affect the overall results.

In conclusion, this study shows that AC angle dimensions can be significantly influenced by $\mathrm{Nd}$ :YAG LPI in European eyes, offering potential protection against acute angle closure. UBM examination is a viable and non-invasive tool for documentation and quantification of the angle morphology. This study assessed the short-term effects of LPI in European eyes. More long-term effects of LPI could be the subject of future research.

\section{References}

1 Quigley HA, Broman AT. The number of people with glaucoma worldwide in 2010 and 2020. Br J Ophthalmol 2006; 90(3): 262-267.

2 Seah SK, Foster PJ, Chew PT, Jap A, Oen F, Fam HB et al. Incidence of acute primary angle-closure glaucoma in Singapore. An island-wide survey. Arch Ophthalmol 1997; 115(11): 1436-1440.

3 Foster PJ, Baasanhu J, Alsbirk PH, Munkhbayar D, Uranchimeg D, Johnson GJ. Glaucoma in Mongolia. A population-based survey in Hovsgol province, northern Mongolia. Arch Ophthalmol 1996; 114(10): 1235-1241.

4 Foster PJ, Johnson GJ. Glaucoma in China: how big is the problem? Br J Ophthalmol 2001; 85(11): 1277-1282.

5 Alsagoff Z, Aung T, Ang LP, Chew PT. Long-term clinical course of primary angle-closure glaucoma in an Asian population. Ophthalmology 2000; 107(12): 2300-2304.

6 Gazzard G, Friedman DS, Devereux JG, Chew P, Seah SK. A prospective ultrasound biomicroscopy evaluation of changes in anterior segment morphology after laser iridotomy in Asian eyes. Ophthalmology 2003; 110(3): 630-638.

7 Nolan WP, Foster PJ, Devereux JG, Uranchimeg D, Johnson GJ, Baasanhu J. YAG laser iridotomy treatment for primary angle closure in East Asian eyes. Br J Ophthalmol 2000; 84(11): 1255-1259.

8 Dada T, Mohan S, Sihota R, Gupta R, Gupta V, Pandey RM. Comparison of ultrasound biomicroscopic parameters after laser iridotomy in eyes with primary angle closure and primary angle closure glaucoma. Eye 2007; 21(7): 956-961. 
9 Fleck BW, Dhillon B, Khanna V, Fairley E, McGlynn C. A randomised, prospective comparison of Nd:YAG laser iridotomy and operative peripheral iridectomy in fellow eyes. Eye 1991; 5(Part 3): 315-321.

10 Friedman DS. Who needs an iridotomy? Br J Ophthalmol 2001; 85(9): 1019-1021.

11 Ishikawa H, Liebmann JM, Ritch R. Quantitative assessment of the anterior segment using ultrasound biomicroscopy. Curr Opin Ophthalmol 2000; 11(2): 133-139.

12 He M, Foster PJ, Johnson GJ, Khaw PT. Angle-closure glaucoma in East Asian and European people. Different diseases? Eye 2006; 20(1): 3-12.

13 He M, Friedman DS, Ge J, Huang W, Jin C, Lee PS et al. Laser peripheral iridotomy in primary angle-closure suspects: biometric and gonioscopic outcomes: the Liwan Eye Study. Ophthalmology 2007; 114(3): 494-500.

14 Marraffa M, Marchini G, Pagliarusco A, Perfetti S, Toscano A, Brunelli $C$ et al. Ultrasound biomicroscopy and corneal endothelium in Nd:YAG-laser iridotomy. Ophthalmic Surg Lasers 1995; 26(6): 519-523.

15 Weinreb RN, Friedman DS. Angle Closure and Angle Closure Glaucoma. Kugler Publications: The Hague, The Natherlands, 2006.

16 Foster PJ, Buhrmann R, Quigley HA, Johnson GJ. The definition and classification of glaucoma in prevalence surveys. Br J Ophthalmol 2002; 86(2): 238-242.

17 Pavlin CJ, Harasiewicz K, Eng P, Foster FS. Ultrasound biomicroscopy of anterior chamber structures in normal and glaucomatous eyes. Am J Ophthalmol 1992; 113. 381-389.

18 Kaushik S, Kumar S, Jain R, Bansal R, Pandav SS, Gupta A. Ultrasound biomicroscopic quantification of the change in anterior chamber angle following laser peripheral iridotomy in early chronic primary angle closure glaucoma. Eye 2007; 21(6): 735-741.

19 Ang LP, Aung T, Chew PT. Acute primary angle closure in an Asian population: long-term outcome of the fellow eye after prophylactic laser peripheral iridotomy. Ophthalmology 2000; 107(11): 2092-2096.

20 Radhakrishnan S, Rollins AM, Roth JE, Yazdanfar S, Westphal V, Bardenstein DS et al. Real-time optical coherence tomography of the anterior segment at $1310 \mathrm{~nm}$. Arch Ophthalmol 2001; 119(8): 1179-1185.

21 Radhakrishnan S, Goldsmith J, Huang D, Westphal V, Dueker DK, Rollins AM et al. Comparison of optical coherence tomography and ultrasound biomicroscopy for detection of narrow anterior chamber angles. Arch Ophthalmol 2005; 123(8): 1053-1059.

22 Tello C, Liebmann J, Potash SD, Cohen H, Ritch R. Measurement of ultrasound biomicroscopy images: intraobserver and interobserver reliability. Invest Ophthalmol Vis Sci 1994; 35(9): 3549-3552.

23 Urbak SF, Pedersen JK, Thorsen TT. Ultrasound biomicroscopy. II. Intraobserver and interobserver reproducibility of measurements. Acta Ophthalmol Scand 1998; 76(5): 546-549. 